\title{
ISO ammonia line absorption reveals a layer of hot gas veiling Sgr B2*
}

\author{
C. Ceccarelli ${ }^{1,2}$, J.-P. Baluteau ${ }^{3}$, M. Walmsley ${ }^{4}$, B. M. Swinyard ${ }^{5}$, E. Caux ${ }^{6}$, S. D. Sidher ${ }^{5}$, P. Cox ${ }^{7}$, \\ C. Gry ${ }^{3,8}$, M. Kessler ${ }^{9}$, and T. Prusti ${ }^{9}$ \\ 1 Observatoire de Bordeaux, BP 89, 33270 Floirac, France \\ ${ }^{2}$ Laboratoire d'Astrophysique, Observatoire de Grenoble - BP 53, 38041 Grenoble Cedex 09, France \\ 3 Laboratoire d'Astrophysique de Marseille, CNRS \& Université de Provence, BP 8, 13376 Marseille Cedex 12, \\ France \\ 4 Osservatorio di Arcetri, 5 L. go E. Fermi, 50125 Firenze, Italy \\ 5 Rutherford Appleton Laboratory, Chilton, Didcot OX1 3RH, UK \\ ${ }^{6}$ CESR CNRS-UPS, BP 4346, 31028 Toulouse Cedex 04, France \\ 7 Institut d'Astrophysique Spatiale, 91405 Orsay, France \\ 8 ISO Data Center, Astrophysics Division, Space Science Department of ESA, Villafranca del castillo, \\ PO Box 50727, I28080 Madrid, Spain \\ 9 Space Science Department of ESA, ESTEC, Postbus 299, 2200 AG Noordwijk, The Netherlands
}

Received 25 September 2001 / Accepted 20 November 2001

\begin{abstract}
We report the first results of the unbiased spectral high resolution survey obtained towards Sgr B2 with the Long Wavelength Spectrometer on board ISO. The survey detected more than one hundreds lines from several molecules. Ammonia is the molecule with the largest number (21) of detected lines in the survey. We detected $\mathrm{NH}_{3}$ transitions from levels with energies from 45 to $500 \mathrm{~cm}^{-1}$. The detected transitions are from both para and ortho ammonia and metastable and non-metastable levels. All the ammonia lines are in absortion against the FIR continuum of Sgr B2. With such a large number of detected lines in such a large range of energy levels, we could very efficiently constrain the main parameters of the absorbing gas layer. The gas is at $(700 \pm 100) \mathrm{K}$ and has a density lower than $10^{4} \mathrm{~cm}^{-3}$. The total $\mathrm{NH}_{3}$ column density in the layer is $(3 \pm 1) \times 10^{16} \mathrm{~cm}^{-2}$, equally shared between ortho and para ammonia. Given the derived relatively high gas temperature and ammonia column density, our observations support the hypothesis previously proposed of a layer of shocked gas between us and Sgr B2. We also discuss previous observations of far infrared line absorption from other molecules, like $\mathrm{H}_{2} \mathrm{O}$ and $\mathrm{HF}$, in the light of this hot absorbing layer. If the absorption is done by the hot absorbing layer rather than by the warm envelope surrounding Sgr B2, as was previously supposed in order to interpret the mentioned observations, the derived $\mathrm{H}_{2} \mathrm{O}$ and $\mathrm{HF}$ abundances are one order of magitude larger than previously estimated. Yet, the present $\mathrm{H}_{2} \mathrm{O}$ and $\mathrm{HF}$ observations do not allow one to disentangle the absorption from the hot layer against the warm envelope. Our conclusions are hence that care should be applied when interpreting the absorption observations in Sgr B2, as the hot layer clearly seen in the ammonia transitions may substantially contribute to the absorption.
\end{abstract}

Key words. infrared: ISM: lines and bands - ISM: individual: Sgr B2 - stars: formation - techniques: spectroscopic

\section{Introduction}

For the conditions prevailing in molecular clouds, either harboring newly born stars or not, the gas cooling in

Send offprint requests to: C. Ceccarelli,

e-mail: Ceccarelli.Cecilia@observ.u-bordeaux.fr

* ISO is an ESA project with instruments funded by ESA

Member States (especially the PI countries: France, Germany, The Netherlands and the United Kingdom) and with the participation of ISAS and NASA. those regions is primarily due to atomic and/or molecular line emission in the Far InfraRed (FIR) to millimeter wavelength range. Light molecules, mainly hydrides, for example $\mathrm{H}_{2} \mathrm{O}$ or $\mathrm{OH}$ which are believed to be the dominant gas coolants in many situations (e.g. Neufeld et al. 1995), have their rotational lines in the FIR, which is heavily absorbed by the Earth's atmosphere. Conversely, heavier molecules such as $\mathrm{H}_{2} \mathrm{CO}$ have their low-lying lines at millimeter to submillimeter wavelengths, which can in part be observed from ground. Molecules with 
intermediate mass like CO have transitions in the entire millimeter to FIR wavelength range. Depending on the gas temperature and density, either the low-lying or highlying lines of the same molecule can be excited, so that multi-frequency studies of the same molecule are commonly used to constraint these parameters together with the column density of the species. Finally, observations by different chemical species provide the relative abundance of them, namely the chemical composition of the gas. In short, knowing which atom/molecule emits or absorbs at what wavelength is of paramount importance to study the gas thermal balance and the physical, dynamical and chemical structure of the gas in molecular clouds.

It is of no surprise then that several unbiased surveys of spectral line emission in the millimeter atmospheric windows have been carried out for a number of sources to study how the physical and chemical conditions change in different environments. The massive star formation regions of Sgr B2, Orion and other few high luminosity protostars have been the favorite targets of these studies in the bands accessible from ground-based telescopes (e.g. Schilke et al. 1997; Nummelin et al. 1998; Schilke et al. 2001). In contrast, a spectral survey in the $350 \mathrm{GHz}$ band exists of only one low luminosity protostar, namely IRAS 16293-2422 (Blake et al. 1994). However, the spectral interval at frequencies higher than $1 \mathrm{THz}$ remained largely unexplored because of the atmosphere opacity at these wavelengths.

The launch of the Infrared Space Observatory (ISO: Kessler et al. 1996) in 1995 allowed us for the first time to explore the FIR interval without the obstacle of telluric absorption. During the ISO Central Programme we carried out an unbiased spectral survey at the highest possible spectral resolution power $(\sim 8000)$ between 47 and $196 \mu \mathrm{m}$ in the direction of Sgr B2, using the Long Wavelength Spectrometer (LWS: Clegg et al. 1996). More than one hundreds lines have been detected in this survey from several chemical species. Most, if not all, of the detected lines are in absorption. In this paper we report the first results concerning the detection of 21 pure rotational lines from ammonia, the species showing the largest number of detected transitions in the survey. The FIR ammonia lines are all observed in absorption against the strong FIR continuum of Sgr B2, testifying to the presence of a gas layer between us and Sgr B2 that "veils" the source. Since the observed ammonia lines cover a rather large energy level range $\left(\sim 45-500 \mathrm{~cm}^{-1}\right)$ we are able to efficently constrain the characteristics of this absorbing layer, specifically its temperature and density, as well as the $\mathrm{NH}_{3}$ column density. The layout of the paper is the following: after a review of the previous relevant studies of Sgr B2 (Sect. 2) we report the observations (Sect. 3) and the results of the data analysis (Sect. 4). In Sect. 5 we present a model for the intervening absorbing layer and in Sect. 6 we discuss some implications of the presence of this veiling layer, specifically with respect to previous published absorption line observations towards Sgr B2.

\section{Source characteristics}

\subsection{The Sgr B2 complex}

Sgr B2 is a massive $\left(\sim 7 \times 10^{6} M_{\odot}\right)$ and dense molecular cloud, $\sim 45 \mathrm{pc}$ in diameter, at a distance of $8.5 \mathrm{kpc}$ from the Sun and relatively close to the Galactic Center (projected distance $\sim 100 \mathrm{pc}$; Lis \& Goldsmith 1989) ${ }^{1}$. It harbors many high luminosity protostars, making it one of the brightest known galactic IR sources (Thronson \& Harper 1986). It is composed of more than fifty compact and ultra-compact HII regions (Martin \& Downes 1972) and several hot cores (Vogel et al. 1987). The three brightest clusters, named N (north), M (main) and S (south) respectively, are roughly aligned in a N-S direction and contain around $5 \%$ of the total cloud mass (Scoville et al. 1975).

These clusters are embedded in a relatively dense and warm $(\sim 100 \mathrm{~K})$ envelope which extends about $5 \mathrm{pc}$ in the N-S direction and $2.5 \mathrm{pc}$ in the $\mathrm{W}-\mathrm{E}$ direction (e.g. Hüttemeister et al. 1993): the density of the warm envelope is $\sim 3 \times 10^{5} \mathrm{~cm}^{-3}$ at $1 \mathrm{pc}\left(=24^{\prime \prime}\right)$ and decreases roughly as the inverse of the distance from the center. The kinetic temperature of the gas in the warm envelope is higher than the dust temperature at a distance larger than $1 \mathrm{pc}$, implying that the gas and dust are thermally decoupled and the gas is not heated by the central source radiation field, at least not in a "canonical" way: dissipation by turbulent motions is perhaps responsible for the increased gas temperature (De Vicente et al. 1997). According to de Vincente et al. a hot $(\sim 100 \mathrm{~K})$ ring of $\sim 3 \mathrm{pc}$ in radius surrounds the Sgr B2M and Sgr B2N complexes. The ring has a depth of 1.4 parsec and it is likely to be the interface between the molecular cloud and the ionized bubbles created by the massive protostars of Sgr B2.

Finally, a hotter $(\geq 300 \mathrm{~K})$ and tenuous $\left(\sim 10^{3} \mathrm{~cm}^{-3}\right)$ gas surrounds the entire Sgr B2 complex. This has mainly been observed in the absorption lines of a variety of molecular species against the background of the radiation from the HII regions and hot dust within the molecular cloud discussed above. We will refer to this component seen mainly in absorption as the hot envelope. In the next subsection, we give a brief description of past studies of this component of Sgr B2.

\subsection{Absorption line measurements towards Sgr B2}

Molecular absorption lines towards Sgr B2 have been known since the late 1960s when both OH (Palmer \& Zuckerman 1967) and $\mathrm{H}_{2} \mathrm{CO}$ (Snyder et al. 1969) were discovered in absorption at a velocity close to that of Sgr B2 emission lines. It soon became clear (Fomalont \& Weliachew 1973; Bieging 1976) that the observed absorption was against the background of the free-free emission emanating from the ionized gas in Sgr B2. Ammonia was also found in absorption (Winnewisser et al. 1979)

\footnotetext{
${ }^{1}$ Other authors report different estimates for the distance, e.g. $7.1 \mathrm{kpc}$ in Reid et al. (1988).
} 
although one required angular resolutions of better than an arc minute to distinguish the absorption from the emission lines originating in the surrounding molecular clouds. One interesting characteristic of these results was the extremely high column densities inferred ranging from $10^{17} \mathrm{~cm}^{-2}$ for $\mathrm{OH}$ to roughly $2 \times 10^{15} \mathrm{~cm}^{-2}$ for both formaldehyde and ammonia. This implied either high hydrogen column densities or higher abundances than typical values in molecular clouds.

Another interesting feature of the ammonia observations was that the temperature inferred for the absorbing gas from the $\mathrm{NH}_{3}$ data was larger than $100 \mathrm{~K}$. This clearly implied physical conditions greatly different from that in local molecular clouds. The absorption was moreover extended on the scale of a few parsecs since it was seen towards both Sgr B2 M and N (projected separation $1.5 \mathrm{pc}$ ). This was confirmed by VLA studies (Vogel et al. 1987; Gaume \& Claussen 1990) which showed a relatively smooth absorption over the region where compact HII regions were present. The absorption was moreover observed essentially only in the metastable $\mathrm{NH}_{3}$ inversion transitions suggesting moderate excitation conditions (localised absorption in contrast was seen in some nonmetastable lines). Taken together, these data suggested that an extended moderate density but high temperature region extending over a few parsec was responsible for the absorption. In fact, the formaldehyde studies (Wilson et al. 1980) suggested densities in the absorbing gas of the order of $10^{4} \mathrm{~cm}^{-3}$. However, the problem of explaining the high temperatures remained and in fact was compounded when measurements of high excitation metastable lines up to $(J, K)=(14,14)$ (Wilson et al. 1982; Hüttemeister et al. 1995) suggested that there might be a component with a temperature as high as $600 \mathrm{~K}$.

This posed (and still poses) the problem of providing a heating source for the extended cloud. Various solutions to this problem have been offered including $\mathrm{C}$-shocks (Flower et al. 1995) and a high cosmic ray flux (e.g. Wilson et al. 1982). Here, we merely note that this hot absorbing layer has unique characteristics within our Galaxy. An example of this is the fact that $\mathrm{NH}_{2}$ (van Dishoeck et al. 1993) was found in absorption towards Sgr B2 and nowhere else in the Galaxy. The abundance of $\mathrm{NH}_{2}$ in the absorbing layer is similar to that of $\mathrm{NH}_{3}$. It seems likely therefore that the Sgr B2 absorbing layers have unusual chemical characteristics and likely that the unusual abundances and high temperature are linked. This together with the detection of FIR and submillimeter lines of water (Cernicharo et al. 1997; Neufeld et al. 2000) and species such as $\mathrm{SiO}$ in absorption (Greaves et al. 1992) perhaps argues in favor of the shock hypothesis, but the question is far from settled.

\section{Observations and data reduction}

The observations were performed with the ISO telescope pointed at $\alpha=17^{\mathrm{h}} 47^{\mathrm{m}} 21^{\mathrm{s}} .7$ and $\delta=-28^{\circ} 23^{\prime} 14^{\prime \prime}$ (J2000). This position, including the whole Sgr B2M source, was chosen in order to avoid the northern component Sgr B2N within the LWS field of view (about $80^{\prime \prime}$ diameter; Swinyard et al. 1998). The complete spectrum of Sgr B2M, between 47 and $196 \mu \mathrm{m}$, has been recorded with the high spectral resolution power mode $(\Delta \lambda / \lambda \sim 8000)$ of the LWS, AOT L03, using the LWS Fabry-Perot (FP). A partial description of the operation and calibration of this AOT can be found in Swinyard et al. (1998) and in the ISO/LWS Handbook (Gry et al. 2001).

The whole spectrum was observed using 31 different AOTs between ISO revolutions 476 (6 March 1997) and 509 (8 April 1997) with a total target dedicated time (TDT) of 47 hours. Additional observations were also performed (6 additional AOTs), between ISO revolutions 836 (28 February 1998) and 849 (14 March 1998), for 10 hours more of TDT, in order to get a higher signal to noise ratio in the 167-194 $\mu \mathrm{m}$ range. Each mini-scan within any observation were repeated three times (four or six times for the additional observations) in order to get good confidence in the data, with a sampling interval of a quarter of the resolution spectral element. The integration time per individual data point was alawys $0.5 \mathrm{~s}$. FP observations with the LWS are implemented as a series of miniscans (over a spectral range of about $0.2 \mu \mathrm{m}$ for the AOT L03). For each miniscan, the LWS grating is set to a fixed position and the selected FP is scanned over a range of gap (separation between the FP meshes) values. At the end of this series of miniscans, the grating is moved to a different position and the next series of miniscans begins. Although the grating adjustment is made for only one detector (the "prime" detector) at a time, data are recorded for all detectors together; the nine other detectors are the "non-prime" detectors. Each miniscan samples the grating response profile. When perfectly adjusted, for the prime detector, the miniscan covers the top of the grating profile, i.e. with the highest overall transmission of the LWS. For non-prime detectors the miniscans are located anywhere on the grating profile, depending on the respective separation between the grating position central wavelength and the wavelength of the FP main order when at mid gap range. The LWS transmission in these cases are, following the occurence, between zero and the maximum value. In this paper we used all the available observations from prime and non-prime detectors, for each group of $\mathrm{NH}_{3}$ lines, in those cases where the transmission is at least one half of the maximum value. A more detailed description of this procedure can be found in Baluteau et al. (in preparation).

The data presented here were processed using the OffLine-Processing pipeline version 7 . However, in order to reduce the prime and non-prime data in the same way, only the first step of the pipeline processing was used: the outputs of this step (Standard Processed Data, or SPD) contains scientific data still in engineering units, i.e. FP gap voltage instead of wavelength and detector photocurrent instead of calibrated flux. Most of the instrumentspecific peculiarities are nevertheless removed and some basic calibrations are performed: construction of ramps, 
removal of unusable readouts, first level deglitching, illuminator flashes processing.

The dedicated LIA procedure FPWAVE was used to convert the FP gap voltage into wavelength, with correction applied to provide the observed wavelength in the heliocentric frame. The two LWS FPs (FPS for $47-70 \mu \mathrm{m}$, and FPL for $70-196 \mu \mathrm{m}$ ) wavelength calibration was performed in flight using a number of astronomical spectral lines of known wavelengths. The calibration appears to be accurate to less than half a resolution element, i.e. about $11 \mathrm{~km} \mathrm{~s}^{-1}$, but the relative uncertainty, for a given AOT, is considered to be much lower $\left(\leq 2.5 \mathrm{~km} \mathrm{~s}^{-1}\right.$; Gry et al. 2001) and allows, in particular, a good determination of the wavelength separation between $\mathrm{NH}_{3}$ metastable and non-metastable line components.

The following steps were performed. First, remaining glitches were removed carefully miniscan by miniscan. Second, the continuum emission level, outside the main spectral features, was fitted using a quadratic curve over each miniscan. Third, a spectrum with flux density normalized to unity was produced. At this step a good knowledge of the dark signal (dark current plus straylight) was required: an estimate of the dark signal, for each part of the spectrum, was performed by looking at non-prime observations of Sgr B2 where the FP and the grating position correspond to close to zero transmission. Last, all prime and non-prime data for each group of $\mathrm{NH}_{3}$ lines were mixed together and weighted by their signal to noise ratios.

The continuum level of the FP data was calibrated against observations of the same line of sight with the LWS in the grating mode (AOT L01) performed during ISO revolution 287. As Sgr B2 is a very strong infrared source the LWS "strong source correction" (see Leeks et al. 2001) was applied to the grating spectrum. We estimate the flux uncertainty to be less than $20 \%$ for the major part of the Sgr B2 spectrum.

\section{Results}

Figure 1 shows a selection of our data. The principal result is that we have detected low-lying transitions with a metastable lower level such as $(3,2) \mathrm{s}-(2,2)$ a with intensities of the order of $30 \%$ of the continuum whereas higher lying lines such as $(8,7)$ s- $(7,7)$ a are roughly an order of magnitude weaker. A surprising result is the clear detection of lines with a non-metastable lower level such as $(4,2) \mathrm{s}^{-}(3,2) \mathrm{a}$ and $(4,1) \mathrm{s}-(3,1) \mathrm{a}$ (clearly detected). The corresponding inversion transitions are not seen in absorption in the single dish radio data (see e.g. Hüttemeister et al. 1993) and we conclude that the FIR lines have advantages relative to radio observations for the purpose of examining the populations of such non-metastable excited levels. Nevertheless, the general feature of the radio data that metastable lines are in absorption and much stronger than non-metastable transitions is reflected also in the FIR observations.
Table 1 lists the $35 \mathrm{NH}_{3}$ lines in the wavelength range covered by our observations. Of these, we detected 21 lines, all in absorption against the continuum. In particular, the lowest (in our wavelength range) 13 lines with metastable lower levels (lower energy levels between 50 and $500 \mathrm{~cm}^{-1}$ ) were detected. Of the remaining 8 lines with non-metastable lower levels, two are from ortho $\mathrm{NH}_{3}$. All the detected lines are centered around 50 to $70 \mathrm{~km} \mathrm{~s}^{-1}$ LSR velocity, consistent with the result from radio observations that the main absorption is at $65 \mathrm{~km} \mathrm{~s}^{-1}$.

As the LWS FP line profile for features in absorption is still practically unknown, we did not use any instrumental profile to fit the data to extract the line fluxes. The line fluxes given in Table 1 were obtained by integrating the observed flux density over each line component. The flux uncertainties reported in this table represent only estimated measurements errors (mainly due to errors in the fitted continuum baseline) and do not include systematic and calibration errors. Dividing by the continuum, this in practice means that we determine equivalent widths for the observed lines.

It is likely that the $\mathrm{NH}_{3}$ lines at long wavelengths (low excitations) are optically thick and interpreting the observed equivalent widths requires constructing a "curve of growth" describing the variation of line intensity with lower level column density. In order to do this, one must make assumptions on the line profile (velocity dispersion) in the absorbing layer. We have assumed for this purpose that radio and infrared lines form in the same region. Thus, we used a simplified model for the $\mathrm{NH}_{3}$ lines based on the radio observations of Hüttemeister et al. (1995). Two Gaussian components, separated by $8.6 \mathrm{~km} \mathrm{~s}^{-1}$, both with the same strength and the same FWHM $\left(8.2 \mathrm{~km} \mathrm{~s}^{-1}\right)$, were convolved with the theoretical FP instrumental profile. Their level column densities were varied in order to give the best agreement with the observed data.

The results of this analysis are given in Fig. 2 where we show the $\mathrm{NH}_{3}$ level column densities normalized by the statistical weights as a function of the energy of the lower level. The derived column densities are corrected for the line optical depth effects described above, which are important (more than 10\%) for the six metastable transitions at the longest wavelengths. The $169.997 \mu \mathrm{m}$, $165.601 \mu \mathrm{m}, 127.111 \mu \mathrm{m}$ and $124.651 \mu \mathrm{m}$ lines have optical depth of order of 1.6, while more highly excited lines are optically thin. Higher optical depths could only result if the FIR lines originate in a layer with less velocity dispersion than the radio transitions and this appears to us unlikely.

On this basis, we derive a rotational temperature of $130 \pm 10 \mathrm{~K}$ for the metastable levels and of $310 \pm 100 \mathrm{~K}$ for non-metastable levels. These rotational temperatures have to be considered as lower limits to the kinetic temperature (e.g. Danby et al. 1988). The derived rotational temperature of the metastable transitions is in good agreement with the rotational temperature $(160 \mathrm{~K}$; Huttemeister et al. 1995) derived by the radio observations using metastable inversion transitions with levels in 


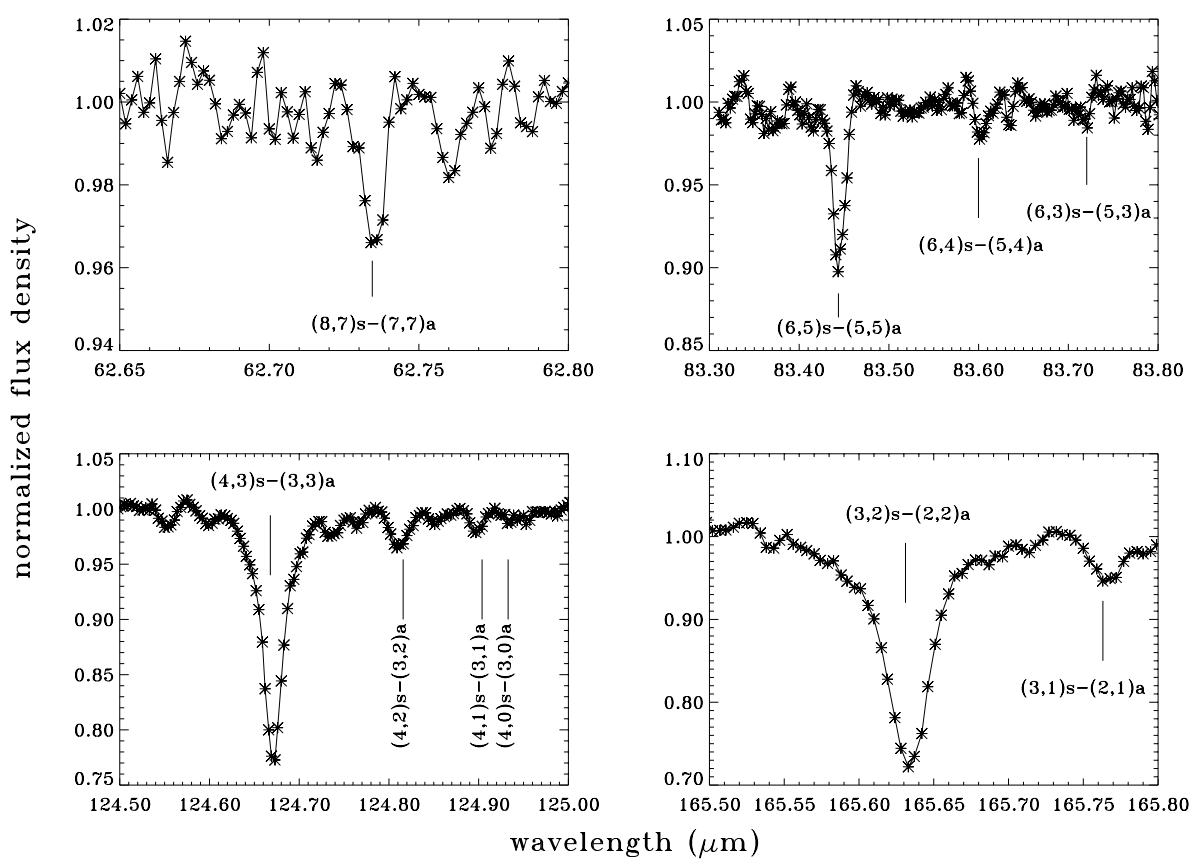

Fig. 1. Selected $\mathrm{NH}_{3}$ lines. Ordinates are the flux normalized to the continuum at each wavelength.

the same energy range, i.e. $50-500 \mathrm{~cm}^{-1}$. Also the column density $\left(N\left(\mathrm{NH}_{3}\right)=2.5 \times 10^{15} \mathrm{~cm}^{-2}\right)$ derived from our rotational diagram of the metastable transitions is in general agreement with the radio estimates $\left(5 \times 10^{15} \mathrm{~cm}^{-2}\right.$; Flower et al. 1995 with an uncertainty of roughly a factor 2 which comes from the excitation temperature of the radio lines themselves). In fact, this rough agreement substantiates the conclusion (see e.g. Flower et al.) that the inversion transitions have excitation temperatures between 3 and $10 \mathrm{~K}$ consistent with formation in a gas of density $10^{3}-10^{4} \mathrm{~cm}^{-3}$.

\section{Modeling}

As described in Sect. 2, Sgr B2 is a complex region and it is not certain, given the relatively large beam of ISO-LWS $\left(\sim 80^{\prime \prime}\right)$, that the layer of gas seen in absorption at radio wavelengths is identical with that giving rise to our observed FIR lines. Indeed, it is unlikely in the strict sense that this is the case since at $\mathrm{cm}$ wavelengths, one observes absorption against the free-free radiation from the compact HII regions embedded in Sgr B2 whereas in the FIR, the observed absorption is against the background of the hot dust in the molecular cloud surrounding those HII regions. In principle moreover, the FIR background itself has a varying extent over the LWS wavelength range and hence the layer being sampled varies from transition to transition. Nevertheless, the radio data indicate that the absorbing layer has an extent of at least $40^{\prime \prime}$ - the separation of Sgr B2M and $\mathrm{N}$ - and we conclude that it is a reasonable approximation to ignore these differences and assume one is "seeing" the same gas at all wavelengths. One should bear in mind however that the detection of FIR absorption as well as the high rotation temperature in transitions with non-metastable lower levels may be a sign of the limitations of this approach. Another result to be borne in mind is that the radio data themselves show evidence of absorption in levels as high as $(14,14)$ (roughly $2000 \mathrm{~K}$ above ground) and thus there is clearly hot (at least $500 \mathrm{~K}$ ) gas present in the radio absorbing layer.

In general however, the simplest interpretation of our data is that the FIR absorption lines originate in the same hot gas observed in the radio transitions and is attributed to shocked gas between us and Sgr B2 (Flower et al. 1995). It seems reasonable that this holds both for transitions with a metastable and with a non-metastable lower level and that the FIR radiation from Sgr B2 itself is responsible for populating non-metastable levels. Thus one has to do with a hot low density medium subjected to a relatively strong FIR radiation field. In the next section, we present a model based on these general premises.

\subsection{Model description}

The sketch of the model adopted for the interpretation of the ammonia data is shown in Fig. 3. A FIR source, Sgr B2M, illuminates a slab of absorbing material: the distance between the slab and the FIR source is dist, while the distance between the slab and the observer is assumed to be $8.5 \mathrm{Kpc}$. The intensity $I_{1}$ of a line (either in emission or absorption) is given by the usual expression (e.g. Tielens \& Hollenbach 1985, Eq. (B1)):

$I_{\mathrm{l}}=\frac{\Omega_{\mathrm{s}}}{2 \pi} \int_{0}^{z} \mathrm{~d} z^{\prime} n_{\mathrm{u}} A_{\mathrm{u}, \mathrm{l}} \beta_{\mathrm{u}, \mathrm{l}}\left(1-\frac{P}{S}\right)$

where $\Omega_{\mathrm{S}}$ is the solid angle of the absorbing slab, $n_{\mathrm{u}}$ is the number density of the molecules in the upper level 
Table 1. List of $\mathrm{NH}_{3}$ lines in the observed wavelength range. The central wavelength, $F W H M$ (in the lines detected at more than $4 \sigma$ ), measured flux with the statistical error in parenthesis, energy of the lower level and the upper and lower states of the transition are in the first, second, third, fourth and fifth columns respectively. The uncertainty in the flux does not include the dark current uncertainty, typically about $5 \%$, and the absolute calibration uncertainty that is $\sim 10 \%$ of the flux.

\begin{tabular}{|c|c|c|c|c|}
\hline $\begin{array}{c}\lambda \\
(\mu \mathrm{m})\end{array}$ & $\begin{array}{c}F W H M \\
(\mu \mathrm{m})\end{array}$ & $\begin{array}{c}\text { Flux } \\
\left(10^{-12} \mathrm{erg} \mathrm{s}^{-1} \mathrm{~cm}^{-2}\right)\end{array}$ & $\begin{array}{c}E_{\text {low }} \\
\left(\mathrm{cm}^{-1}\right)\end{array}$ & Transition \\
\hline 55.814 & 0.005 & $\leq 3$ & 477 & $(9,8) \mathrm{s}-(8,8) \mathrm{a}$ \\
\hline 56.340 & & $0.5(0.3)$ & 478 & $(9,8) \mathrm{a}-(8,8) \mathrm{s}$ \\
\hline 62.729 & 0.019 & $1.8(0.4)$ & 374 & $(8,7) \mathrm{s}-(7,7) \mathrm{a}$ \\
\hline 63.378 & 0.006 & $2.0(0.4)$ & 374 & $(8,7) \mathrm{a}-(7,7) \mathrm{s}$ \\
\hline 71.610 & 0.007 & $12.4(1.2)$ & 282 & $(7,6) \mathrm{s}^{-}-(6,6) \mathrm{a}$ \\
\hline 71.770 & & $\leq 4$ & 324 & $(7,5) \mathrm{s}-(6,5) \mathrm{a}$ \\
\hline 72.440 & 0.012 & $14.7(1.5)$ & 284 & $(7,6) \mathrm{a}-(6,6) \mathrm{s}$ \\
\hline 72.526 & & $0.8(0.5)$ & 325 & $(7,5) \mathrm{a}-(6,5) \mathrm{s}$ \\
\hline 83.434 & 0.011 & $18.0(1.8)$ & 204 & $(6,5) \mathrm{s}-(5,5) \mathrm{a}$ \\
\hline 83.592 & & $2.0(1.0)$ & 237 & $(6,4) \mathrm{s}-(5,4) \mathrm{a}$ \\
\hline 83.712 & & $\leq 3$ & 263 & $(6,3) \mathrm{s}-(5,3) \mathrm{a}$ \\
\hline 84.546 & 0.021 & $9.6(3.0)$ & 206 & $(6,5) \mathrm{a}-(5,5) \mathrm{s}$ \\
\hline 84.623 & & $\leq 9$ & 239 & $(6,4) \mathrm{a}-(5,4) \mathrm{s}$ \\
\hline 84.682 & & $\leq 9$ & 265 & $(6,3) \mathrm{a}-(5,3) \mathrm{s}$ \\
\hline 99.952 & 0.013 & $49.0(3.8)$ & 139 & $(5,4) \mathrm{s}-(4,4) \mathrm{a}$ \\
\hline 100.107 & 0.011 & $3.8(0.6)$ & 165 & $(5,3) \mathrm{s}-(4,3) \mathrm{a}$ \\
\hline 100.215 & & $1.4(0.7)$ & 183 & $(5,2) \mathrm{s}-(4,2) \mathrm{a}$ \\
\hline 100.280 & 0.012 & $\leq 2$ & 194 & $(5,1) \mathrm{s}-(4,1) \mathrm{a}$ \\
\hline 101.536 & 0.021 & $39.0(2.0)$ & 140 & $(5,4) \mathrm{a}-(4,4) \mathrm{s}$ \\
\hline 101.599 & & $2.9(0.6)$ & 166 & $(5,3) \mathrm{a}-(4,3) \mathrm{s}$ \\
\hline 101.645 & & $\leq 2$ & 184 & $(5,2) \mathrm{a}-(4,2) \mathrm{s}$ \\
\hline 101.673 & & $\leq 4$ & 195 & $(5,1) \mathrm{a}-(4,1) \mathrm{s}$ \\
\hline 101.682 & & $\leq 4$ & 199 & $(5,0) \mathrm{a}-(4,0) \mathrm{s}$ \\
\hline 124.651 & 0.024 & $56.0(2.8)$ & 86 & $(4,3) \mathrm{s}-(3,3) \mathrm{a}$ \\
\hline 124.799 & 0.023 & $3.6(1.4)$ & 104 & $(4,2) \mathrm{s}-(3,2) \mathrm{a}$ \\
\hline 124.887 & & $1.8(0.9)$ & 115 & $(4,1) \mathrm{s}-(3,1) \mathrm{a}$ \\
\hline 124.916 & & $\leq 2$ & 119 & $(4,0) \mathrm{s}-(3,0) \mathrm{a}$ \\
\hline 127.111 & 0.019 & $54.0(3.0)$ & 86 & $(4,3) \mathrm{a}-(3,3) \mathrm{s}$ \\
\hline 127.156 & & $\leq 3$ & 104 & $(4,2) \mathrm{a}-(3,2) \mathrm{s}$ \\
\hline 127.184 & & $\leq 2$ & 115 & $(4,1) \mathrm{a}-(3,1) \mathrm{s}$ \\
\hline 165.601 & 0.024 & $58.0(5.0)$ & 45 & $(3,2) \mathrm{s}-(2,2) \mathrm{a}$ \\
\hline 165.733 & & $3.8(1.1)$ & 56 & $(3,1) \mathrm{s}-(2,1) \mathrm{a}$ \\
\hline $169.971^{a}$ & & $49.0(6.0)$ & 45 & $(3,2) \mathrm{a}-(2,2) \mathrm{s}$ \\
\hline $169.991^{a}$ & & & 55 & $(3,1) \mathrm{a}-(2,1) \mathrm{s}$ \\
\hline $169.996^{a}$ & & & 119 & $(3,0) \mathrm{a}-(2,0) \mathrm{s}$ \\
\hline
\end{tabular}

${ }^{a}$ The $(3,2) \mathrm{a}-(2,2) \mathrm{s},(3,1) \mathrm{a}-(2,1) \mathrm{s}$ and $(3,0) \mathrm{a}-(2,0) \mathrm{s}$ transitions are merged.

of the transition, $A_{\mathrm{u}, 1}$ is the spontaneous emission coefficient and $\beta_{\mathrm{u}, \mathrm{l}}$ is the probability that the photons escape absorption. $S$ is the source function and $P$ is the observed intensity of the continuum emission (per unit solid angle) at the wavelength of the transition. Equation (1) implicitly assumes that the ratio $P / S$ is constant over the solid angle. For absorption lines, when the source function and the stimulated emission can be neglected, Eq. (1) gives the usual relation between the line equivalent width and the column density (e.g. Spitzer 1968). When however the source function and the stimulated emission cannot a priori be neglected, one has to use Eq. (1) and to solve the 


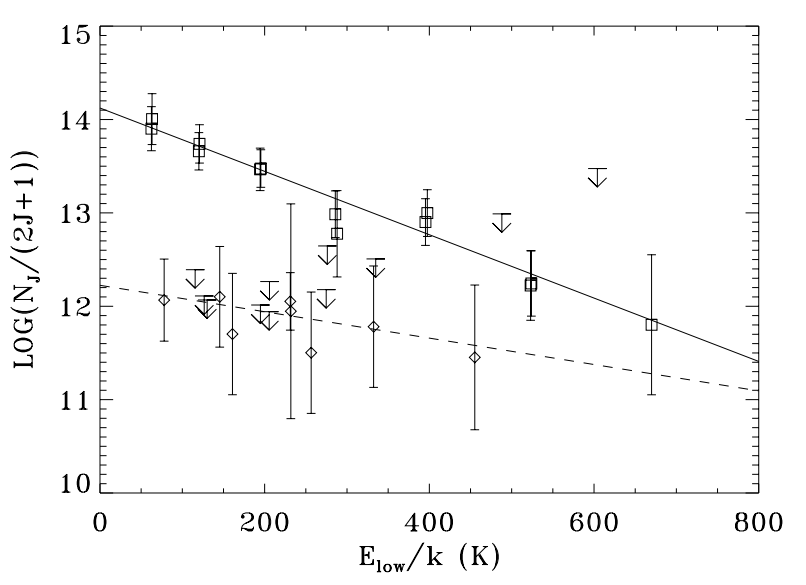

Fig. 2. $\mathrm{NH}_{3}$ level column densities normalized by the statistical weights $(2 J+1)$ as function of the energy of the lower level energy $E_{\text {low }}$. Squares refer to metastable lines and diamonds to non-metastable lines. Arrows indicate upper limits of the nonmetastable lines. The errors bars include also the calibration uncertainty $(\sim 10 \%)$ plus the dark current uncertainty $(\sim 5 \%)$. The solid and dashed lines are the best fit to the metastable and non-metastable line observations respectively. The relevant rotational temperatures are $130 \mathrm{~K}$ and $310 \mathrm{~K}$, and the $\mathrm{NH}_{3}$ column densities are $2.5 \times 10^{15} \mathrm{~cm}^{-1}$ and $8.8 \times 10^{13} \mathrm{~cm}^{-1}$ for the metastable and non-metastable transitions respectively (see text).

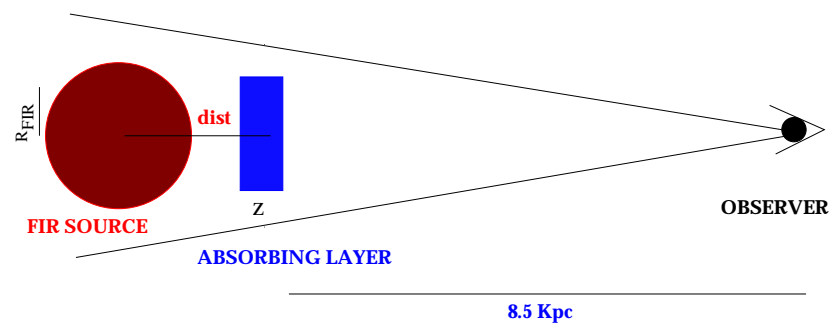

Fig. 3. Sketch of the model: an absorbing layer of depth $z$ lies between us and the FIR source Sgr B2M. Note that the layer may extend more than the FIR source or ISO beam. Here we assume that the $\mathrm{NH}_{3}$ line emission from this part of the layer is negligible.

statistical equilibrium equations for the level populations to compute $n_{\mathrm{u}}$ and $\beta_{\mathrm{u}, 1}$ :

$n_{\mathrm{u}} \sum_{\mathrm{l}}\left(A_{\mathrm{u}, 1} \beta_{\mathrm{u}, \mathrm{l}}+B_{\mathrm{u}, \mathrm{l}} P W+C_{\mathrm{u}, \mathrm{l}}\right)=$
$\sum_{\mathrm{l}} n_{\mathrm{l}}\left(A_{\mathrm{l}, \mathrm{u}} \beta_{\mathrm{l}, \mathrm{u}}+B_{\mathrm{l}, \mathrm{u}} P W+C_{\mathrm{l}, \mathrm{u}}\right)$

where $C$ are the collision coefficients, $B$ the absorption coefficients and $W$ is the geometrical dilution factor of the continuum emitted by the FIR source. This latter parameter accounts for the internal intensity that the $\mathrm{NH}_{3}$ molecules see at a typical location inside the slab:

$W=\frac{1}{2}\left(1-\sqrt{1-\left(\frac{R_{\mathrm{FIR}}}{\text { dist }}\right)^{2}}\right)$ where $R_{\text {FIR }}$ is assumed to be $0.8 \mathrm{pc}$, obtained using 8.5 Kpc for the distance of Sgr B2 and 40" for the observed diameter of the FIR source (Goldsmith et al. 1990) at all considered wavelengths. This is a rough approximation of the real situation as is the fact that the continuum emission $P$ is modeled as a grey body at $31 \mathrm{~K}$ that reproduces the observed spectrum (e.g. Cernicharo et al. 1997). The escape probability $\beta$ is function of the optical depth of the line and we used the relation valid in a planar slab (De Jong et al. 1980):

$\beta=\frac{1-\exp (-3 \tau)}{3 \tau}$.

Our LVG model computes the first 50 levels of the ortho and para ammonia respectively. The two forms are treated as independent species. The $\mathrm{NH}_{3}$ levels and A coefficients were taken from the JPL database (http://spec.jpl.nasa.gov/home.html). The collisional coefficients of the first 17 levels of ortho and para $\mathrm{NH}_{3}$ are those by Danby et al. (1988). Extrapolation for the higher energy levels has been computed following the approximation described in Flower et al. (1995). Finally, we assumed a line $F W H M$ of $15 \mathrm{~km} \mathrm{~s}^{-1}$, as measured in the radio lines.

\subsection{Model results}

The absorption computed by the LVG model depends on five parameters: the density and temperature of the gas, the $\mathrm{NH}_{3}$ column density, the dilution factor $W$, and the solid angle subtended at the earth of the absorbing slab (which may be smaller than the ISO-LWS beam solid angle). The line ratios do not depend on the size of the absorbing slab and we therefore use first the observed flux ratios to constrain the other four parameters.

We ran a series of models with the density varying between $10^{3}$ and $3 \times 10^{7} \mathrm{~cm}^{-3}$, the temperature varying between 30 and $1000 \mathrm{~K}$, the $\mathrm{NH}_{3}$ column density varying between $10^{15}$ and $10^{17} \mathrm{~cm}^{-2}$, and $W$ varying between 0.001 and 0.5 . The results of the modeling are shown in Fig. 4, where we report four selected line ratios as function of the $\mathrm{NH}_{3}$ column density for $W=0.1$, and parametrized by the gas density and temperature. The lower panels show the computed ratio of the lowest and highest observed metastable lines, whereas the upper panels show the ratio between a non-metastable and a metastable line.

As discussed in the literature (e.g. Ho \& Townes 1983; Danby et al. 1988) and suggested by the rotation diagram analysis (Fig. 2) the ammonia metastable levels have a relative population populated approximately according to LTE, and hence their relative line strengths depend mostly on the gas temperature and much less on other parameters. Figure 4 shows that the observed metastable line ratios put a stringent lower limit on the gas temperature, $T \geq 500 \mathrm{~K}$, irrespective of the ammonia column density and of the dilution factor $W$. On the other hand, the nonmetastable populations are clearly sub-thermal and thus the observed non-metastable to metastable line ratios are 


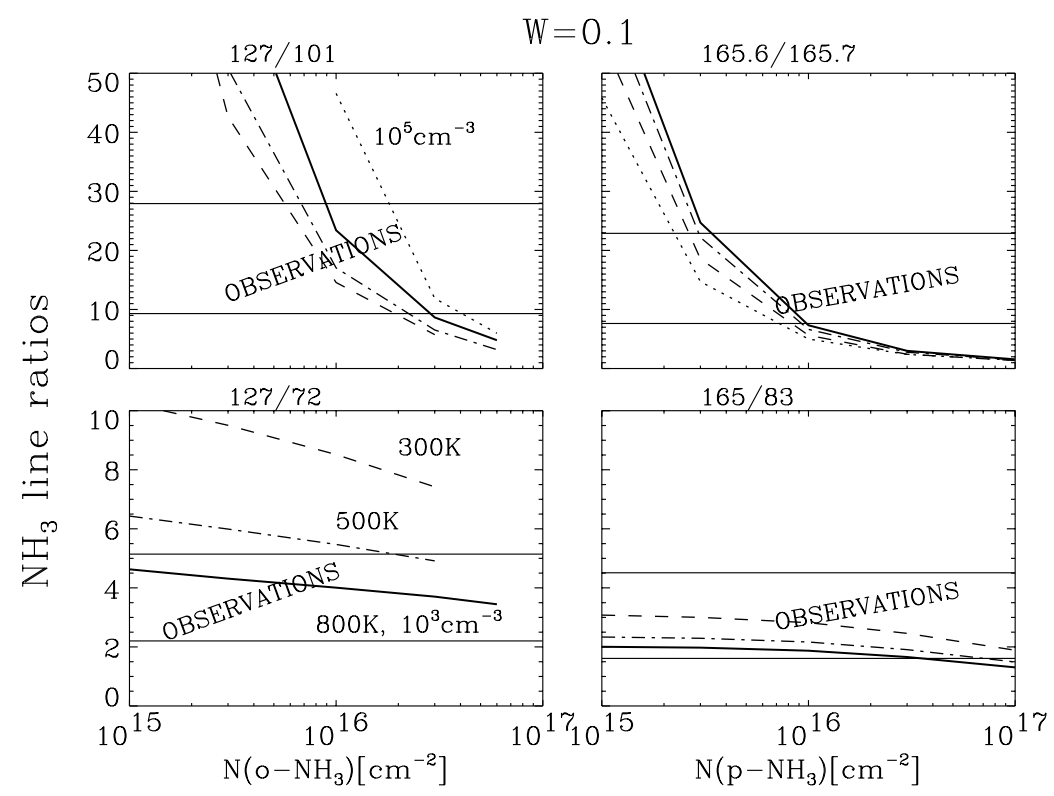

Fig. 4. Line flux ratios: ortho- $\mathrm{NH}_{3}$ lines (left) and para- $\mathrm{NH}_{3}$ lines (right). Upper left panel: $(4,3) \mathrm{a}-(3,3) \mathrm{s}($ at $127.111 \mu \mathrm{m})$ over $(5,3) \mathrm{a}-(4,3) \mathrm{s}($ at $101.599 \mu \mathrm{m})$. Lower left panel:(4, 3)a-(3, 3)s (at $127.111 \mu \mathrm{m})$ over $(7,6) \mathrm{a}-(6,6) \mathrm{s}$ (at $72.440 \mu \mathrm{m})$. Upper right panel: $(3,2) \mathrm{s}-(2,2) \mathrm{s}($ at $165.601 \mu \mathrm{m})$ over $(3,1) \mathrm{s}-(2,1)$ a $($ at $165.733 \mu \mathrm{m})$. Lower right panel: $(3,2) \mathrm{s}-(2,2) \mathrm{s}($ at $165.601 \mu \mathrm{m})$ over $(6,5) \mathrm{s}-(5,5)$ a $($ at $83.434 \mu \mathrm{m})$. Solid lines: model with $n=10^{3} \mathrm{~cm}^{-3}$ and $T=800 \mathrm{~K}$. Dashed lines: models with $n=10^{3} \mathrm{~cm}{ }^{-3}$ and $T=300 \mathrm{~K}$. Dotted-dashed lines: models with $n=10^{3} \mathrm{~cm}^{-3}$ and $T=500 \mathrm{~K}$. Dotted lines: model with $n=10^{5} \mathrm{~cm}^{-3}$ and $T=800 \mathrm{~K}$. Abscissae are the ortho (left panels) and para (right panels) $\mathrm{NH}_{3}$ column densities respectively. Note that dotted and solid lines coincide in the lower panels.

much more sensitive to the gas density. This is not surprising as the critical density of the observed transitions is much higher than the density estimated on the basis of the radio observations. The upper limit on the density from our data is about $10^{5} \mathrm{~cm}^{-3}$, and depends slightly on the dilution factor $W$. For lower $W$ values, the curves of the upper panels of Fig. 2 shift towards higher column densities whereas larger $W$ values generate curves shifted toward lower $\mathrm{NH}_{3}$ column densities.

We also have explored the full parameter space in $W$ and $N\left(\mathrm{NH}_{3}\right)$. Figure 5 shows the $\chi^{2}$ value, namely the sum of the squared differences between the observations and the model predictions, as function of the $\mathrm{o}-\mathrm{NH}_{3}$ column density and $W$. The best fit of the data is obtained with $W=0.15 \pm 0.05$, corresponding to dist $=(1.15 \pm 0.15) \mathrm{pc}$, and $N\left(\mathrm{o}-\mathrm{NH}_{3}\right)=(1.5 \pm 0.5) \times 10^{16} \mathrm{~cm}^{-2}$. Note that these values indicate a "conservative" square of the possible range that $W$ and $N\left(\mathrm{o}-\mathrm{NH}_{3}\right)$ can assume, but the lowest $\chi^{2}$ is a triangle (Fig. 5). From the average ratio between observed fluxes and model predictions, we then derive the absorbing slab size to be $\Delta \theta=30^{\prime \prime}$. We note that this is formally in contradiction with the radio result that the absorbing slab has to be $40^{\prime \prime}$ in extent but conclude that given the uncertainties of the geometry of both the slab and the FIR source, this difference is not significant. Furthermore, the $30^{\prime \prime}$ sizes derived for the hot layer are by definition a lower limit to the real extent, as the layer could be more extended than the FIR source.

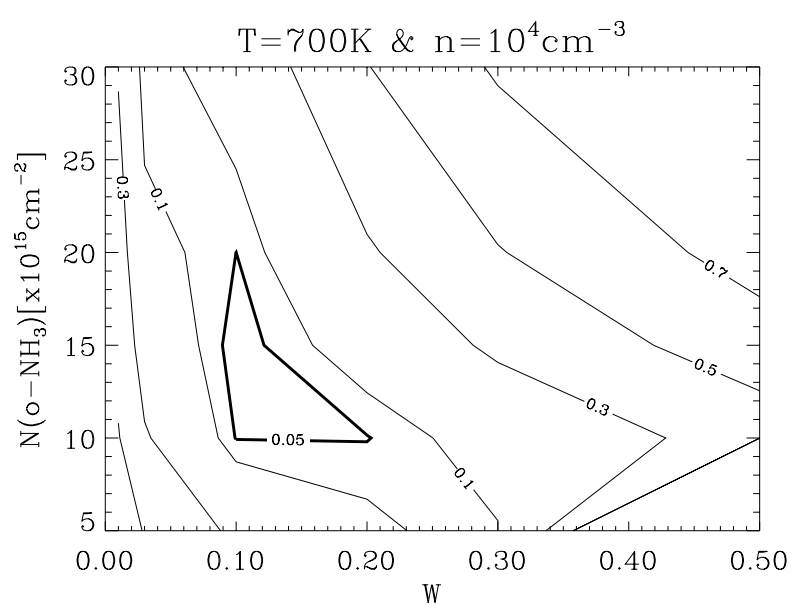

Fig. 5. $\chi^{2}=\frac{1}{N-1} \sum_{1}^{N}(\text { Observation }- \text { Model })^{2}$ as function of the o- $\mathrm{NH}_{3}$ column density and the $W$ parameter. The plot is obtained with $T=700 \mathrm{~K}$ and $n=10^{4} \mathrm{~cm}^{-3}$, but it is essentially the same for temperatures larger than $600 \mathrm{~K}$ and densities smaller than $10^{5} \mathrm{~cm}^{-3}$.

Finally, we constrained the $\mathrm{p}-\mathrm{NH}_{3}$ column density by taking the values derived by the $\mathrm{o}-\mathrm{NH}_{3}$ observations, $\left(W=0.15, T=700 \mathrm{~K}, n=10^{4} \mathrm{~cm}^{-3}\right.$ and $\left.\Delta \theta=30^{\prime \prime}\right)$ and minimizing the $\chi^{2}$ value as a function of the $\mathrm{p}-\mathrm{NH}_{3}$ column density. We found $N\left(\mathrm{p}-\mathrm{NH}_{3}\right)=(1.5 \pm 0.5) \times 10^{16} \mathrm{~cm}^{-2}$, the uncertainty coming from the uncertainty on $W$ and $\Delta \theta$. Notice that our observations put a stringent constraint 
Table 2. The best fit model parameters.

\begin{tabular}{|c|c|}
\hline \hline Temperature & $(700 \pm 100) \mathrm{K}$ \\
density & $\leq 10^{5} \mathrm{~cm}^{-3}$ \\
$W$ & $0.15 \pm 0.05$ \\
dist & $(1.15 \pm 0.15) \mathrm{pc}$ \\
extent & $30^{\prime \prime}$ \\
$N\left(\mathrm{o}-\mathrm{NH}_{3}\right)$ & $(1.5 \pm 0.5) \times 10^{16} \mathrm{~cm}^{-2}$ \\
$N\left(\mathrm{p}-\mathrm{NH}_{3}\right)$ & $(1.5 \pm 0.5) \times 10^{16} \mathrm{~cm}^{-2}$ \\
$\mathrm{o} / \mathrm{p}$ & 1 \\
$N\left(\mathrm{NH}_{3}\right)$ & $(3.0 \pm 1.0) \times 10^{16} \mathrm{~cm}^{-2}$ \\
\hline \hline
\end{tabular}

on the ortho to para ratio, which results a value equal to unity, within $20 \%$. Hence we do not confirm the suggestion by Flower et al. (1995) that $\mathrm{o} / \mathrm{p}$ is less than the statistical value of 1 .

In conclusion, our FIR observations are explained by an absorbing layer of gas with the parameters summarized in Table 2. The comparison of the predictions of the best fit model with the observations are shown in Fig. 6 as a function of the lower level energy. The agreement is reasonable though we note that the model predictions for the para transitions above $300 \mathrm{~K}$ are consistently too low. This may suggest that the temperature is even higher than the assumed $700 \mathrm{~K}$.

As a final remark, we note that no FIR CO lines have been detected in the survey. The lack of CO emission detection can be used to give an upper limit to the density of the gas. Assuming a $\mathrm{NH}_{3}$ abundance equal to $10^{-6}$ (see the discussion in Sect. 6.2), and a CO abundance in the layer of $10^{-4}$, the lack of FIR CO emission implies a density lower than $3 \times 10^{3} \mathrm{~cm}^{-3}$. Given the uncertainty on the actual CO column density and the emitting region sizes, we conservatively think that the density has indeed to be $\leq 10^{4} \mathrm{~cm}^{-3}$, consistent with what has previously been discussed.

\section{Discussion}

We found that the absorption at the $\mathrm{NH}_{3}$ transitions is caused by a layer of gas at a temperature of $\sim 700 \mathrm{~K}$ and with a density of $\sim 10^{4} \mathrm{~cm}^{-3}$. The layer covers $\sim 30^{\prime \prime}$ of the ISO-LWS beam, is at a distance of $\sim 1 \mathrm{pc}$ from the FIR continuum source and has a total $\mathrm{NH}_{3}$ column density of $\sim 3 \times 10^{16} \mathrm{~cm}^{-2}$, equally shared between the ortho and para forms. Our observations therefore confirm the previous findings (see the discussion in Sect. 2.2) and complement them, better constraining some key parameters: the gas temperature and density, the $\mathrm{NH}_{3}$ column density and the ortho-to-para ratio.

Having improved constraints on the physical parameters of the absorbing layer, we are in a better position to understand the origin of this hot gas on the one hand and the implication of its presence for the interpretation of previous absorption observations on the other hand.

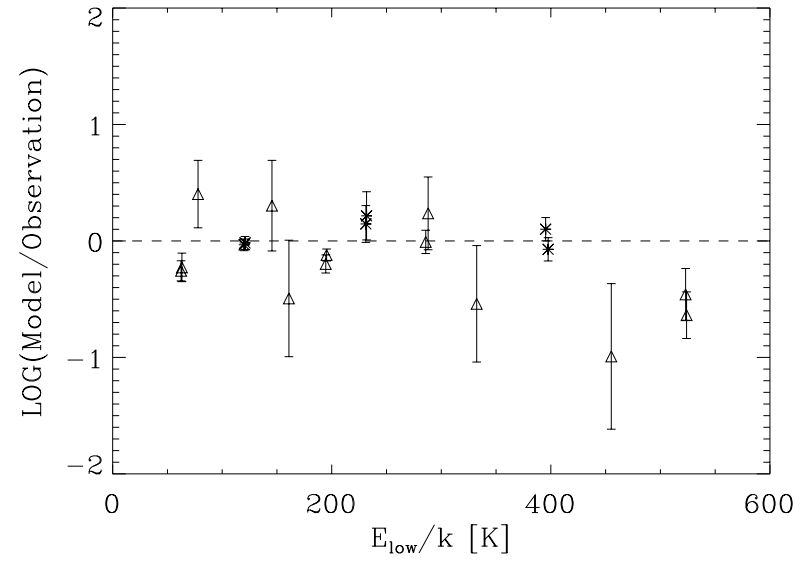

Fig. 6. The ratio between the best fit model (Table 2) predictions and the observations as function of the lower level energy. Asterisks show the o- $\mathrm{NH}_{3}$ transitions, triangles show the $\mathrm{p}-\mathrm{NH}_{3}$ transitions.

We discuss in some detail these two issues, starting with the latter.

\subsection{Previous absorption observations}

In this section, we revise the interpretation of some previous absorption observations towards Sgr B2. As mentioned in Sect. 2.2, water has been detected in absorption in several transitions and in particular in the ground state line (Zmuidzinas et al. 1995; Neufeld et al. 2000), and in the $2{ }_{12}-1_{01}$ line (Cernicharo et al. 1997) (both transitions in the $\mathrm{H}_{2}^{16} \mathrm{O}$ and $\mathrm{H}_{2}^{18} \mathrm{O}$ isotope forms). As emphasized by the authors, the interpretation of the observed $\mathrm{H}_{2} \mathrm{O}$ absorption is complicated by the variety of velocity components showing absorption along the line of sight to Sgr B2. In the cited papers, the authors interpreted the absorption observed around $65 \mathrm{~km} \mathrm{~s}^{-1}$ as due to the warm envelope surrounding Sgr B2 (see Sect. 2).

Although an accurate modeling of the water spectrum is beyond the scope of this article (and we leave it to a forthcoming paper), we wish to consider here the possibility that the absorption is caused by the hot gas detected by our $\mathrm{NH}_{3}$ observations. Using the parameters derived from our $\mathrm{NH}_{3}$ observations (Table 2) and leaving the water column density as a free parameter, we can reproduce approximatively the $\mathrm{H}_{2}^{18} \mathrm{O} 1_{10}-1_{01}$ and $2_{12}-1_{01}$ observations simultaneously with a $\mathrm{H}_{2}^{18} \mathrm{O}$ column density equal to $1 \times 10^{15} \mathrm{~cm}^{-2}$. Assuming $\mathrm{H}_{2}^{16} \mathrm{O} / \mathrm{H}_{2}^{18} \mathrm{O}=250$ (e.g. Neufeld et al. 2000), this gives a water column density of $2.5 \times 10^{17} \mathrm{~cm}^{-2}$ in the absorbing layer, i.e. about ten times larger than the estimate reported by Zmuidzinas et al. (1995) and Neufeld et al. (2000).

This is an extremely interesting result, that is relevant both to the interpretation of the absorption observations in general (as discussed in Neufeld et al. 2000) and to the interpretation of the origin of the layer itself. Flower et al. (1995) suggested that a shock is responsible for the heating 
of the gas layer and developed an accurate model for such a shock. Their model accounted reasonably well for the radio ammonia observations (from which they estimated a $\mathrm{NH}_{3}$ column density of $\sim 2 \times 10^{16} \mathrm{~cm}^{-2}$ ), but predicted a water column density of $\sim 7 \times 10^{17} \mathrm{~cm}^{-2}$ which is much too large compared to the Zmuidzinas et al. estimates. With our new estimate, the difference is much smaller and the shock model is thus reinforced. We will further discuss this issue in the next section.

Other species have been observed in absorption against the continuum of Sgr B2. It is certainly worth verifying whether the $\mathrm{NH}_{3}$ absorbing layer can account also for these observations and what it would imply. In particular, atomic oxygen (Baluteau et al. 1997) and ionized carbon (Cox et al. 2000) have been reported in absorption towards Sgr B2. However high resolution observations show that the absorption in this case occurs around $0 \mathrm{~km} \mathrm{~s}^{-1}$ and it is therefore not associated with Sgr B2 itself but is rather due to the material between us and the galactic center (Vastel et al. in preparation). Our data exclude absorption in the FIR ammonia lines at these velocities, although radio observations show a hot component absorbing $\mathrm{NH}_{3}$ at $0 \mathrm{kms}^{-1}$ (Gardner et al. 1988), maybe associated with Sgr B2 itself. Probably this component has a too low $\mathrm{NH}_{3}$ column density to be detected by our observations.

Another interesting case is the HF $2 \leftarrow 1$ transition at $121.7 \mu \mathrm{m}$ that has been observed in absorption around $65 \mathrm{~km} \mathrm{~s}^{-1}$, i.e. in the gas associated with the Sgr B2 complex (Neufeld et al. 1997). Neufeld et al. considered the case where the absorption is due to the warm envelope surrounding Sgr B2 and derived a HF abundance of $3 \times 10^{-10}$. The relatively low value derived for the $\mathrm{HF}$ abundance led Neufeld et al. to suggest that fluorine is substantially depleted in Sgr B2. Lacking other detected transitions it is difficult to assess the parameters of the absorbing layer. If it originates in the $\mathrm{NH}_{3}$ absorbing layer rather than in the warm envelope, the HF column density would be $\sim 4 \times 10^{14} \mathrm{~cm}^{-2}$ and $\mathrm{HF} / \mathrm{NH}_{3} \sim 0.01$. Assuming a $\mathrm{NH}_{3}$ abundance of $10^{-6}$ (see next section) would imply a fluorine abundance equal to $10^{-8}$, i.e. 30 times larger than the abundance derived by Neufeld et al., reducing the inferred depletion to a much smaller value.

Finally, we wish to remark that this analysis shows the possibility that the absorption observed in the $\mathrm{H}_{2} \mathrm{O}$ and $\mathrm{HF}$ molecules is due, at least in part, to the hot layer. Yet, given the available data, it is not possible to argue that this is definitively the case, as low-lying transitions are certainly affected by absorption from colder material in the line of sight. We want therefore to caution against the over-interpretation of such data.

\subsection{The chemical composition and origin of the layer}

As already remarked by Flower et al. (1995), the relatively high temperature and $\mathrm{NH}_{3}$ column density of the absorbing layer point towards a shock origin. Following their reasoning, the average ammonia abundance in the layer must be around $10^{-6}$, to have a plausible hydrogen column density. Then, assuming that the water and HF observed line absorption originates in the hot layer too (as discussed in the previous section) would imply a water abundance of $\sim 10^{-5}$ and a HF abundance of $\sim 10^{-8}$. Both abundances would be close to their relevant elemental abundances (oxygen and fluorine respectively), consistent with the hypothesis that a shock has substantially eroded the grain mantles, releasing their constituents into the gas phase. The mass of the shocked gas in the ISOLWS beam is larger than $500 M_{\odot}$ assuming a $\mathrm{NH}_{3}$ abundance of $10^{-6}$, and the corresponding thermal energy is $2.5 \times 10^{46}$ ergs. This is a large amount of hot gas whose origin presumably is linked to the rapid star formation in Sgr B2. We note that such a hot shocked layer is unusual in our galaxy and hence may be related to a short-lived event. In summary, ammonia, water and HF absorption line observations are all consistent with the hypothesis that a hot gas created by a shock and associated with Sgr B2 is the cause of the observed absorption lines.

\section{Conclusions}

We report the observations of 21 ammonia pure rotational lines, all of them detected in absorption against the FIR continuum of Sgr B2. We show that the absorption is due to a layer of hot $(\sim 700 \mathrm{~K})$ and tenuous $\left(\sim 10^{4} \mathrm{~cm}^{-3}\right)$ gas between us and Sgr B2. The total ammonia column density in the layer is $\sim 3 \times 10^{16} \mathrm{~cm}^{-2}$, shared equally between the ortho and para forms. Given the derived high temperature of the gas, our observations support the suggestion by Flower et al. (1995) that the layer is probably formed by shocked gas. Our results also offer a solution to the discrepancy between the shock model predictions of the water abundance and previous observational determinations (Zmuidzinas et al. 1995; Neufeld et al. 2000; Cernicharo et al. 1997). This discrepancy is resolved if the observed water absorption lines do not originate in the warm envelope, as previously supposed, but rather in the hot shocked layer of gas. We also showed that the HF absorption reported by Neufeld et al. (1997) can be explained as well as being due to the hot layer: in this case we derived a HF abundance 30 times larger than that derived by Neufeld et al. assuming that the absorption originates in the warm envelope. In summary, we wish to caution that care should be taken in the analysis of the lines in absorption in the direction of Sgr B2, as the same may happen to other observed molecular species, especially when only a few lines from the same molecule are observed. This may change some of the claims about the chemistry in the cloud surrounding Sgr B2, which, being sometimes the only place where these species are observed, is of particular relevance.

Acknowledgements. We wish to warmly thank Pierre Valiron for providing us the electronic file for the $\mathrm{NH}_{3}$ collisional coefficients, Claire Rist for useful discussions about the collisional coefficients and the entire LWS team, whose work made these observations possible. We wish also to thank J. Cernicharo, our referee, whose insightful comments helped to improve the text. 


\section{References}

Baluteau, J.-P., Cox, P., Cernicharo, J., et al. 1997, A\&A, 322, L25

Bieging, J. H. 1976, A\&A, 51, 289

Blake, G. A., van Dishoeck, E. F., Jansen, D. J., et al. 1994, ApJ, 428, 680

Cernicharo, J., Lim, T., Cox, P., et al. 1997, A\&A, 323, L25

Clegg, P. E., Ade, P. A. R., Armand, C., et al. 1996, A\&A, 315, L38

Cox, P., Roelfsema, P. R., Baluteau, J.-P., et al. 2000, in The Universe as seen by ISO, ed. P. Cox, \& M. F. Kessler, ESA SP-427

Danby, G., Flower, D. R., Valiron, P., Schilke, P., \& Walmsley, C. M. 1988, MNRAS, 235, 229

De Jong, T., Dalgarno, A., \& Boland, W. 1980, A\&A, 91, 68

de Vicente, P., Martin-Pintado, J., \& Wilson 1997, A\&A, 320, 957

Flower, D. R., Pineau des Forêts, G., \& Walmsley, M. 1995, A\&A, 294, 815

Fomalont, E. B., \& Weliachew, L. N. 1973, ApJ, 181, 781

Gardner, F. F., Boes, F., \& Winnewisser, G. 1988, A\&A, 196 207

Gaume, R. A., \& Claussen, M. J. 1990, ApJ, 351, 538

Goldsmith, P. F., Lis, D. C., Hills, R., \& Lasenby, J. 1990, ApJ, 350,186

Greaves, J. S., White, G. J., Ohishi, M., Hasegawa, T., \& Sunada, K. 1992, A\&A, 260, 381

Gry, C., Swinyard, B. M., Andrew, A., et al. 2001, ISO-LWS Handbook ESA Publications, FAI-99-077/Dc version 1.2

Ho, P. T. P., \& Townes, C. H. 1983, ARA\&A, 21, 239

Huttemeister, S., Wilson, T. L., Henkel, C., \& Mauesberger, R. 1993, A\&A, 276, 445

Huttemeister, S., Wilson, T. L., Mauesberger, R., et al. 1995, A\&A, 294, 667

Kessler, M. F., Steinz, J. A., Anderegg, M. E., et al. 1996, A\&A, 315, L27

Leeks, S. J., Swinyard, B. M., \& Clegg, P. E. 2001, Proc. The calibration legacy of the ISO Mission, ed. L. Metcalfe, \& M. F. K. Kessler
Lis, D. C., \& Goldsmith, P. F. 1989, ApJ, 337, 704

Lis, D. C., Keene, J., Phillips, T. G., et al. 2001, ApJ, in press Martin, A. H. M., \& Dowens, D. 1972, ApJ, 11, 219

Neufeld, D. A., Lepp, S., \& Melnick, G. J. 1995, ApJS, 100, 132

Neufeld, D. A., Zmuidzinas, J., Schilke, P., \& Phillips, T. G. 1997, ApJ, 488, L141

Neufeld, D. A., Ashby, M. L .N., Bergin, E. A., et al. 2000, ApJ, 539, L111

Nummelin, A., Bergman, P., Hjalmarson, A., et al. 1998, ApJS, 117,427

Palmer, P., \& Zuckerman, B. 1967, ApJ, 148, 727

Reid, M. J., Schneps, M. H., Moran, J. M., et al. 1988, ApJ, 330,809

Schilke, P., Groesbeck, T. D., Blake, G. A., \& Phillips, T. G. 1997, ApJS, 108, 301

Schilke, P., Benford, D. J., Hunter, T. R., Lis, D. C., \& Phillips, T. G. 2001, ApJS, 132, 281

Scoville, N. Z., Solomon, P. M., \& Penzias, A. A. 1975, ApJ, 201, 352

Snyder, L. E., Buhl, D., Zuckerman, B., \& Palmer, P. 1969, Phys. Rev. Lett., 22, 67

Swinyard, B. M., Burgdorf, M. J., Clegg, P. E., et al. 1998, SPIE, 3354, 888

Spitzer 1968, Physical processes in the interstellar medium (ed. J. Wiley \& Sons, Inc.)

Tielens, X., \& Hollenbach, D. 1985, ApJ, 291, 722

Thronson, H. A., \& Harper, D. A. 1986, ApJ, 300, 396

van Dishoeck, E. F., Jansen, D. J., Schilke, P., \& Phillips, T. G. 1993, ApJ, 416, L83

Vogel, S. N., Genzel, R., \& Palmer, P. 1987, ApJ, 316, 243

Wilson, T. L., Walmsley, C. M., Henkel, C., Pauls, T., \& Mattes, H. 1980, A\&A, 91, 36

Wilson, T. L., Ruf, K., Walmsley, C. M., et al. 1982, A\&A, 115,185

Winnewisser, G., Churchwell, E., \& Walmsley, C. M. 1979, A\&A, 72, 215

Zmuidzinas, J., Blake, G. A., Carlstrom, J., Keene, J., \& Miller, D. 1995, ApJ, 447, L125 